\title{
Relevance of Geriatric Assessment in Older Patients With Colorectal Cancer
}

\author{
Lore Decoster, ${ }^{1}$ Leen Vanacker, ${ }^{1}$ Cindy Kenis, ${ }^{2,3}$ Hans Prenen, ${ }^{4}$ Erik Van Cutsem, ${ }^{4}$ \\ Jacques Van Der Auwera, ${ }^{1,5}$ Ellen Van Eetvelde, ${ }^{6}$ Katrien Van Puyvelde, ${ }^{7}$ \\ Johan Flamaing, ${ }^{3,8}$ Koen Milisen, ${ }^{3,9}$ Jean Pierre Lobelle, ${ }^{10}$ Jacques De Grève, ${ }^{1}$ \\ Hans Wildiers ${ }^{2,11}$
}

\begin{abstract}
With the aging of the population with colorectal cancer, there is a need for a more personalized approach of these older patients. The present study demonstrates that the performance of a geriatric assessment identifies problems and predicts functional decline during treatment as well as chemotherapy-related toxicity. Geriatric assessments should be integrated in the care of older patients with colorectal cancer.

Introduction: This study aims to evaluate the relevance of geriatric assessment (GA) in older patients with colorectal cancer (CRC) and to study functional status (FS) and chemotherapy-related toxicity during treatment. Methods: Patients with $C R C$ aged $\geq 70$ years were evaluated at baseline using a GA. Results were communicated to the treating physician. At 2 to 3 months follow-up, FS was reassessed, and chemotherapy-related toxicity was recorded. Results: A total of 193 patients, with a median age of 77 years, were included. GA was abnormal in $75 \%$ and revealed unknown problems in $40 \%$. Treatment was altered in $37 \%$ based on clinical assessment. GA led to geriatric interventions in 9 patients $(5 \%)$ and additionally influenced treatment in 1 patient. At follow-up $(n=164)$, functional decline was observed in 29 patients (18\%) for activities of daily living (ADL) and in 60 patients (37\%) for instrumental activities of daily living (IADL). Baseline IADL, depression, fatigue, and cognition were predictors for ADL decline, whereas no predictors for IADL decline could be identified. In the 109 patients receiving chemotherapy, stage and baseline fatigue were predictive for grade $3 / 4$ hematologic toxicity, and baseline ADL, fatigue, and nutrition were predictive for grade $3 /$ 4 nonhematologic toxicity. Conclusion: Although GA identified previously unknown problems in more than one-third of older CRC patients, the impact on interventions or treatment decisions was limited. Baseline GA parameters may predict functional decline and chemotherapy-related toxicity. Education of physicians treating older patients with CRC is an essential step in the implementation of GA and subsequent interventions.
\end{abstract}

Clinical Colorectal Cancer, Vol. ., No. - - - @ 2016 Published by Elsevier Inc.

Keywords: Biologic age, Chemotherapy-related toxicity, Elderly, Functional decline, Treatment decision

\section{Introduction}

The prevalence of colorectal cancer (CRC) increases with age. Over $50 \%$ of patients with newly diagnosed CRC are older than 70 years of age. ${ }^{1}$ With the aging of the population, it is expected that the incidence of CRC in this older population will rise further.

L.D. and L.V. contributed equally to this work as first authors.

${ }^{1}$ Department of Medical Oncology, Oncologisch Centrum, UZ Brussel, Brussels, Belgium

${ }^{2}$ Department of General Medical Oncology

${ }^{3}$ Department of Geriatric Medicine

${ }^{4}$ Department of Gastroenterology, University Hospitals Leuven, Leuven, Belgium

${ }^{5}$ Department of Gastroenterology, AZ St Monica, Deurne, Belgium

${ }^{6}$ Department of Surgery, UZ Brussel, Brussels, Belgium

${ }^{7}$ Department of Geriatric Medicine, ASZ, Aalst, Belgium

${ }^{8}$ Department of Clinical and Experimental Medicine
However, these patients are underrepresented in clinical trials ${ }^{2}$ and often understaged and undertreated. ${ }^{3,4}$

Older patients with cancer represent a heterogeneous group with discrepancies between chronologic and biologic age owing to differences in functional and cognitive status and the presence of

\footnotetext{
${ }^{9}$ Department of Public Health and Primary Care, Academic Centre for Nursing and Midwifery, KU Leuven, Leuven, Belgium

${ }^{10}$ Consultant in Statistics, Beernem, Belgium

${ }^{11}$ Department of Oncology, KU Leuven, Leuven, Belgium
}

Submitted: May 18, 2016; Revised: Jul 19, 2016; Accepted: Jul 28, 2016

Address for correspondence: Lore Decoster, MD, Department of Medical Oncology, Oncologisch Centrum, UZ Brussel, Laarbeeklaan 101, 1090 Brussels, Belgium

E-mail contact: lore.decoster@uzbrussel.be 


\section{Geriatric Assessment in Colorectal Cancer}

comorbidities and polypharmacy. The Eastern Cooperative Oncology Group (ECOG) performance status (PS) and standard clinical approach fail to evaluate those aspects specifically for older patients with cancer. ${ }^{5}$

Geriatric medicine has developed a multidimensional geriatric assessment $(\mathrm{GA})^{6}$ to identify deficits missed by routine examination. It includes social parameters, functional status (FS) and fall history, cognitive and psychological status, nutritional status, comorbidities and polypharmacy, and uses validated geriatric scales to identify frail patients and subsequently set up an individualized geriatric intervention plan.

In older patients with cancer, GA is feasible at large scale and detects unknown geriatric problems. ${ }^{7,8}$ In addition, GA and subsequent interventions can improve quality of life and seem to be

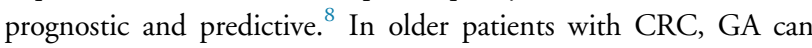
predict treatment-related toxicity and postoperative morbidity. ${ }^{9,10}$

The aim of the present study was to investigate the value of GA in older patients with CRC as well as its influence on cancer treatment decisions. Secondly, the study evaluated the evolution of FS during treatment and the development of chemotherapy-related severe toxicity.

\section{Methods}

\section{Patient Population}

We performed a prospective noninterventional cohort study on GA in older patients with cancer in 6 tumor types (breast cancer, CRC, lung cancer, ovarian cancer, prostate cancer, and hematologic malignancies) in 2 Belgian academic hospitals. ${ }^{7}$ The ethical committees of both participating centers approved the study (protocol number S51815).

Patients 70 years and older with newly diagnosed cancer or cancer progression/relapse were included when a cancer treatment decision had to be made. Disease progression/relapse was defined as progression during treatment or relapse after a treatment-free interval. A written informed consent was obtained from all patients.

For the present study, we performed a subanalysis on the cohort of patients with CRC.

\section{Geriatric Screening and $G A$}

At baseline, a trained health care worker performed a geriatric screening and GA in all patients, as previously described. ${ }^{7}$ Geriatric screening was performed with the Flemish version of the Triage Risk Screening Tool (fTRST) and G8. ${ }^{11-13}$ The GA included: pain assessment, social data, FS by the Katz Activities of Daily Living (ADL) and by the Lawton Instrumental Activities of Daily Living (IADL), fall history during the last year, fatigue assessed by the Mobility Tiredness scale (MOB-T), mental status by the Mini Mental State Examination (MMSE) and Geriatric Depression Scale (GDS-15), nutritional status by the Mini Nutritional AssessmentShort Form (MNA-SF), comorbidities by the Charlson Comorbidity Index (CCI), and a polypharmacy assessment. ${ }^{12}$

A geriatric risk profile was defined as having 2 or more of the following criteria: living alone, $\mathrm{ADL}>6$, IADL $<8$ for females or $<5$ for males, MMSE $<24 / 30$, GDS $>5 / 15$, MNA-SF $<24 /$ 30 , and $\mathrm{CCI} \geq 1$.

The GA results were communicated to the treating physician electronically or on paper prior to the final cancer treatment decisions. It was at the physician's discretion to consult this information for the final cancer treatment plan. If deemed necessary, the initial treatment plan could be modified, and specific interventions could be performed.

Geriatric interventions consisted of referrals to the geriatrician, social worker, physiotherapist, psychologist, dietician, geriatric day clinic, fall clinic, and other.

\section{Questionnaires for the Treating Physician}

After the final treatment decision, the treating physician was interviewed using a predefined questionnaire. First, the questionnaire contained 3 questions on the GA: (1) Were you aware of GA results at the time of treatment decision? (2) Did the GA reveal any new information? and (3) Was any action undertaken to deal with the problems detected by the GA? Second, the questionnaire included 3 main questions on the impact of age and GA on cancer treatment decisions: (1) What would be your oncologic treatment proposal if the patient was 55 years old without comorbidities? (2) Is this different from your treatment proposal for this older patient according to age and standard clinical assessment without information from the GA? If yes, what was your treatment proposal for this patient and why? and (3) Is this different from your current treatment proposal for this patient according to age and standard clinical approach with the knowledge of GA results? If yes, what is your current treatment proposal for this patient and why?

The treatment proposed for a 55-year old patient without comorbidities (answer to question 1) was considered as standard. If the answer to the second question was affirmative, treatment was considered changed based on clinical assessment including age. Only physicians who consulted the GA results before the final treatment decision could answer the third question on treatment decisions. If the answer to this question was affirmative, treatment was considered modified based on GA results.

\section{Functional Decline}

The FS of patients was reassessed 2 to 3 months after the cancer treatment decision by repeating the ADL and IADL. Functional decline on the $\mathrm{ADL}$ was defined as an increase of 2 or more points on the total score, and on IADL as a decrease of 1 or more points on the total score. ${ }^{12}$

\section{Chemotherapy-Related Toxicity}

For patients treated with chemotherapy, grade $3 / 4$ toxicity, according to the common terminology criteria for adverse events, v4.0, was retrospectively recorded. ${ }^{14}$ Hematologic and nonhematologic toxicity were analyzed separately.

\section{Data Analysis}

Data were analyzed using SAS v9.3. For continuous data, mean, median, 95\% confidence intervals (CIs), and range were assessed. For categorical data, frequency and 95\% CI were assessed. Categorical data were compared using the $\chi^{2}$ test.

Determination of predictors of functional decline on the ADL and IADL and of grade $3 / 4$ hematologic and nonhematologic chemotherapy toxicity was performed separately using logistic regression. Univariate logistic regressions were conducted on the 
functional decline of the ADL and IADL in all patients and on hematologic toxicity and nonhematologic toxicity in patients receiving chemotherapy.

For functional decline, the following baseline categorical variables were used: age (70-74/75-79/ $\geq 80$ years), time point of assessment (new diagnosis vs. disease progression/relapse), stage (I-III vs. IV), ECOG PS (score $0-1$ vs. score $2-4)$, fTRST $(\geq 1)$, G8 $(\leq 14)$, baseline $\operatorname{ADL}(>6)$, and IADL $(<5$ [male]/8 [female]), living situation (living alone), fall history in the past 12 months (reported fall history), fatigue by MOB-T (presence of fatigue), cognition by MMSE $(<24)$, depression by GDS-15 $(\geq 5)$, nutrition by MNA-SF $(\leq 11)$, comorbidities by CCI $(\geq 1)$, polypharmacy $(\geq 5$ different drugs), surgery, radiotherapy, and chemotherapy.

For chemotherapy-related toxicity, surgery, radiotherapy, and chemotherapy were excluded from the analysis. In addition, for nonhematologic toxicity, cognition by MMSE was excluded owing to a complete separation of data points.

Multivariable logistic regressions were conducted on the functional decline of the ADL and IADL and on grade 3/4 hematologic and nonhematologic chemotherapy-related toxicity, using stepwise variable selection and $P$-values to enter and to stay in the model of, respectively, .20 and .15 . Only variables significant in the univariate setting were considered for the multivariable regressions.

\section{Results}

\section{Patient Characteristics}

From October 2009 till July 2011, 937 patients were included of which 193 were patients with CRC (Table 1).

Median age in the CRC cohort was 77 years (range, 70-89 years). The majority (67\%) of patients had stage IV disease.

At follow up, 2 to 3 months after inclusion, the data of 164 patients $(85 \%)$ were available (Figure 1).

\section{Results of Baseline Geriatric Screening and Assessment}

The screening tools fTRST and G8 were abnormal in 156 (81\%) and $145(75 \%)$ patients, respectively (Table 2).

A geriatric risk profile by GA was observed in 144 (75\%) patients. Deficiencies were observed in all GA domains, with risk for malnutrition being the most frequent in $130(67 \%)$ patients. At baseline, 93 patients (48\%) showed dependency on ADL and 111 patients $(58 \%)$ on IADL.

Ninety-six of 145 patients (66\%) with ECOG PS $<2$ and all 47 patients with ECOG PS $\geq 2$ had a geriatric risk profile, according to the GA.

\section{Influence of Clinical Assessment (Including Age) and GA on Cancer Treatment Decisions}

The questionnaire for the treating physician was completed in 183 of 193 patients (95\%).

Treatment was adapted based on clinical assessment in $67 \mathrm{pa}$ tients (37\%; 95\% CI, 30\%-49\%) (Table 3), mostly for systemic therapy including chemotherapy and targeted therapy (60 patients): 6 patients received the standard regimen at reduced dose, $20 \mathrm{pa}-$ tients received a less toxic regimen at standard dose, 4 patients received a less toxic regimen at reduced dose, and 30 patients did not receive systemic therapy.
Table 1 Patients and Clinical Characteristics $(n=193)$

\begin{tabular}{|c|c|c|}
\hline Characteristic & $\mathbf{N}$ & $\%$ \\
\hline \multicolumn{3}{|l|}{ Age, years } \\
\hline Median (range) & 77 (70-89) & \\
\hline \multicolumn{3}{|l|}{ Gender } \\
\hline Female & 73 & 37.8 \\
\hline Male & 120 & 62.2 \\
\hline \multicolumn{3}{|c|}{ Colorectal carcinoma stage } \\
\hline Stage I & 13 & 6.7 \\
\hline Stage ॥ & 25 & 13 \\
\hline Stage III & 25 & 13 \\
\hline Stage IV & 130 & 67.3 \\
\hline \multicolumn{3}{|l|}{ ECOG PS } \\
\hline 0 & 104 & 53.9 \\
\hline 1 & 41 & 21.2 \\
\hline 2 & 23 & 11.9 \\
\hline 3 & 22 & 11.4 \\
\hline 4 & 2 & 1 \\
\hline M & 1 & 0.5 \\
\hline \multicolumn{3}{|l|}{ Treatment } \\
\hline Surgery & 86 & 44.6 \\
\hline Radiotherapy & 18 & 7 \\
\hline Chemotherapy & 109 & 56.5 \\
\hline Adjuvant & 18 & \\
\hline Palliative & 91 & \\
\hline
\end{tabular}

Abbreviation: ECOG PS = Eastern Cooperative Oncology Group Performance Status.

Standard treatment was not given to 9 of 46 patients with a normal GA (20\%; 95\% CI, 8.4\%-31.6\%) and to 58 of 137 patients with an abnormal GA (42\%; 95\% CI, 33.7\%-50.3\%) $(P=.006)$.

\section{Figure 1 Flow Chart of Patient Selection}

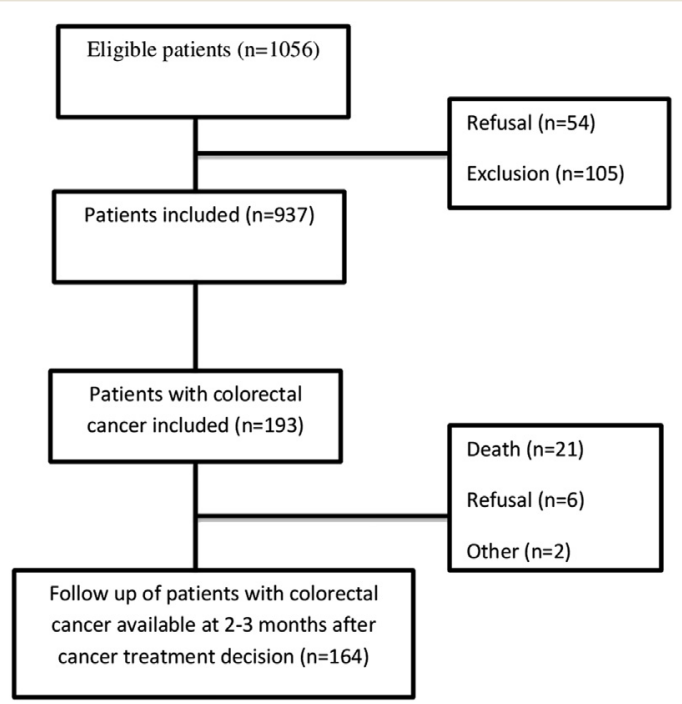




\section{Geriatric Assessment in Colorectal Cancer}

Table 2 Results of the Baseline Geriatric Screening and Assessment

\begin{tabular}{|c|c|c|c|c|}
\hline & Item/Instrument & Score & $\mathbf{N}$ & $\%$ \\
\hline \multirow[t]{4}{*}{ Screening } & fTRST (0-6) & Absence of a geriatric risk profile: score 0 & 37 & 19.2 \\
\hline & & Presence of a geriatric risk profile: score $\geq 1$ & 156 & 80.8 \\
\hline & G8 $(0-17)$ & Absence of a geriatric risk profile: score $>14$ & 48 & 24.9 \\
\hline & & Presence of a geriatric risk profile: score $\leq 14$ & 145 & 75.1 \\
\hline \multicolumn{5}{|l|}{ Geriatric assessment } \\
\hline \multirow[t]{2}{*}{ Social data } & Living situation & Alone & 48 & 24.9 \\
\hline & & Not alone & 145 & 75.1 \\
\hline \multirow[t]{6}{*}{ Functional status } & ADL (6-24) & Independent: score $=6$ & 100 & 51.8 \\
\hline & & Dependent: score $\geq 7$ & 93 & 48.2 \\
\hline & IADL $(0-5 / 8)$ & Independent: score $=8$ (female) or $=5$ (male) & 82 & 42.5 \\
\hline & & Dependent: score $<8$ (female) or $<5$ (male) & 111 & 57.5 \\
\hline & Falls history in the past 12 months & No falls & 135 & 70 \\
\hline & & $\geq 1$ falls & 58 & 30 \\
\hline \multirow[t]{2}{*}{ Fatigue } & MOBT-T (0-6) & No fatigue & 62 & 32.1 \\
\hline & & Presence of fatigue & 131 & 67.9 \\
\hline \multirow[t]{2}{*}{ Cognition } & MMSE $(0-30)$ & Normal cognition: score $\geq 24$ & 177 & 91.7 \\
\hline & & Mild/severe cognitive decline: score $<24$ & 16 & 8.3 \\
\hline \multirow[t]{2}{*}{ Depression } & GDS $(0-15)$ & Not at risk for depression: score $<5$ & 155 & 80.3 \\
\hline & & At risk for depression: score $\geq 5$ & 38 & 19.7 \\
\hline \multirow[t]{2}{*}{ Nutrition } & MNA (0-30) & Normal nutritional status score $\geq 24$ & 85 & 44 \\
\hline & & At risk for malnutrition score $<24$ & 108 & 56 \\
\hline \multirow[t]{2}{*}{ Co-morbidities } & $\mathrm{CCl}(0-37)$ & No comorbidities: score $=0$ & 81 & 42 \\
\hline & & Co-morbidities: score $\geq 1$ & 112 & 58 \\
\hline \multirow[t]{3}{*}{ Polypharmacy } & Number of different drugs & $0-4$ & 106 & 55 \\
\hline & & $\geq 5$ & 82 & 42.4 \\
\hline & & M & 5 & 2.6 \\
\hline
\end{tabular}

Abbreviations: $\mathrm{ADL}=$ activities of daily living; $\mathrm{CCl}=$ Charlson Comorbidity Index; fTRST = Flemish version of the Triage Risk Screening Tool; GA = geriatric assessment; GDS = Geriatric Depression Scale; IADL = instrumental activities of daily living; $\mathrm{M}=$ missing data; MMSE = Mini Mental State Examination; MNA = Mini Nutritional Assessment; MOB-T = Mobility - Tiredness Test.

Before the final treatment decision, physicians actively consulted the GA results for 78 (43\%) patients.

The GA revealed unknown geriatric problems in $40 \%$, mainly concerning fall history $(15 \%)$, risk of depression (11\%), and functional dependency (11\%). In 9 patients $(5 \%)$, the identified problems led to a directed intervention, including consultation of a social worker (1\%), a geriatrician (1.5\%), a psychologist $(0.5 \%)$, and a dietician (2\%).

The results of the GA led to an additional change of treatment in 1 patient: palliative care instead of chemotherapy in a patient with stage IV CRC.

\section{Functional Decline at Follow-Up}

At 2 to 3 months follow-up ( $\mathrm{n}=164), 73$ (45\%) patients were dependent on ADL and 98 (60\%) on IADL.

Functional decline for ADL and IADL was observed in $29(18 \%)$ and 60 patients (37\%), respectively. There was no significant difference in functional decline between the baseline independent and dependent group for ADL $(P=.570)$ and $\operatorname{IADL}(P=.763)$.

Table 4 describes univariate and multivariable analysis in all patients for prediction of decline in ADL and IADL. In multivariate analysis, a decline in ADL was predicted by baseline IADL (odds ratio $[\mathrm{OR}], 0.42)$, fatigue $(\mathrm{OR}, 0.37)$; cognition $(\mathrm{OR}, 0.37)$, and risk of depression (OR, 0.44). No predictors for IADL decline were identified.

\section{Chemotherapy-Related Toxicity at Follow-Up}

A total of 109 patients were treated with systemic therapy, of which 18 were adjuvant and 91 were for advanced disease, including fluorouracil or capecitabin in 20 patients (18\%), combination chemotherapy in 37 patients (34\%), combination of chemotherapy with bevacizumab in 20 patients $(18 \%)$, combination of chemotherapy with anti-epidermal growth factor receptor antibody in 14 patients $(13 \%)$, study protocol in 7 patients $(6 \%)$, and other options in 11 patients (10\%). Patients with advanced stage disease (stage III or IV) more frequently received systemic treatment than patients with early stage disease (stage I or II) (103 of 155 [66.5\%] vs. 6 of $38[15.8 \%]$, respectively $[P<.001])$.

Twenty-seven of 109 (25\%) patients treated with systemic therapy experienced grade $3 / 4$ toxicity. Twenty-one patients demonstrated nonhematologic grade $3 / 4$ toxicity, including gastrointestinal toxicity $(\mathrm{n}=10)$, cardiovascular adverse events $(\mathrm{n}=4)$, stomatitis $(\mathrm{n}=2)$, hand-foot syndrome $(\mathrm{n}=2)$, peripheral neuropathy $(\mathrm{n}=1)$, and dyspnea $(\mathrm{n}=2)$. Hematologic toxicity 


\begin{tabular}{l|c|c|}
\hline Table 3 & $\begin{array}{l}\text { Influence of Clinical Assessment (Including Age) on } \\
\text { Cancer Treatment Decision in Older Patients With } \\
\text { Colorectal Cancer, Based on Answers to the Second } \\
\text { Question on the Questionnaire for the Treating Phy- } \\
\text { sicians (N = 183) }\end{array}$ \\
\hline $\begin{array}{l}\text { Differences in Cancer Treatment Decision } \\
\text { in OIder Versus Younger/Fit Patients }\end{array}$ & N & \% \\
\hline Chemotherapy & 60 & 32.8 \\
\hline Standard schedule at reduced dose & 6 & 3.3 \\
\hline Less toxic schedule at standard dose & 20 & 10.9 \\
\hline Less toxic schedule at reduced dose & 4 & 2.2 \\
\hline No chemotherapy & 30 & 16.4 \\
\hline Surgery & 22 & 12.6 \\
\hline Less extensive surgery & 1 & 0.5 \\
\hline No surgery & 4 & 2.2 \\
\hline Radiotherapy & 8 & 4.5 \\
\hline Less extensive radiotherapy & 4 & 2.2 \\
\hline Radiotherapy instead of surgery/chemotherapy & 1 & 0.5 \\
\hline No radiotherapy & 3 & 1.6 \\
\hline
\end{tabular}

occurred in 6 patients, of which 3 were grade 3 anemia, 2 were febrile neutropenia, and 1 was grade 3 neutropenia.

Table 5 describes univariate and multivariable analysis in patients receiving chemotherapy for prediction of hematologic and nonhematologic grade 3/4 toxicity. For hematologic toxicity, multivariate analysis identified stage of disease $(\mathrm{OR}, 6.60)$ and baseline fatigue $(\mathrm{OR}, 8.15)$ as predictive factors. For nonhematologic toxicity, baseline nutrition (OR, 0.33), fatigue (OR, 0.30), and $\mathrm{ADL}(\mathrm{OR}, 2.62)$ were predictive factors.

\section{Discussion}

Physicians treating CRC are confronted with a growing number of older patients, a group underrepresented in clinical trials. ${ }^{2}$ It is unclear whether treatment regimens that are beneficial for younger patients are also the best choice for the older population, given their heterogeneity in physiologic reserves, comorbidities, FS, and cognition. This heterogeneity provides a major challenge, requiring an individualized and multidisciplinary approach.

For the present study, we performed a subanalysis of older patients with CRC included in a large prospective study evaluating the performance of GA in older patients with cancer. ${ }^{7,12,15}$ In 193 patients with CRC aged $\geq 70$ years, a GA was performed before the final cancer treatment decision.

The current analysis suggests that, in more than one-third (37\%) of older patients with CRC, treatment decisions are influenced by clinical assessment (including age), mainly regarding systemic treatment. This supports previous data that older patients with CRC are less likely to undergo surgery ${ }^{3}$ or to receive systemic treatment both in the adjuvant and palliative settings. ${ }^{4}$ In addition, it seems that the multidisciplinary decision-making regarding chemotherapy for CRC is often made without face-to-face contact between the patient and the discussants, and that chronologic age is a frequent motivation for omission of chemotherapy. ${ }^{4}$ Other patient characteristics such as comorbidity were considered less important. ${ }^{16}$ In the current study, $20 \%$ of patients considered fit by GA did not receive standard treatment. Although different reasons may be responsible, including patient choice, a proportion of our older patients with CRC were probably undertreated. This is in contrast to data demonstrating a similar benefit for fit older and younger patients with CRC for surgery and chemotherapy ${ }^{17}$ and a similar tolerance to commonly used chemotherapy regimens, provided there is adequate supportive care. ${ }^{1}$ On the other hand, 58\% of patients considered at risk by GA did receive standard treatment, which may sometimes be appropriate, providing supportive interventions, but a proportion of patients may also be overtreated because oncologists often overestimate the fitness of older patients with cancer for chemotherapy. ${ }^{5}$ For this reason, the performance of a GA may enhance personalized treatment for older patients with CRC and subsequently prevent under- and overtreatment.

In the current study, 66\% of patients with an ECOG PS $<2$ demonstrated a geriatric risk profile according to the GA. This was confirmed by Jolly et al: of 796 older patients with cancer with a good professional and self-rated Karnofsky PS ( $\geq 80$ ), 69\% had at least 1 GA-identified deficit. ${ }^{18}$ In this heterogeneous population, commonly used PS scales seem inferior to GA for selection of fit from unfit patients, mainly because PS scales are normally assigned using cancer-related FS and not to comorbidities. In addition, GA may predict surgical morbidity ${ }^{19}$ and chemotherapy-related toxicity. $^{20,21}$

In the present study, $75 \%$ of patients demonstrated an abnormal GA, with deficiencies observed in all domains, mostly for nutrition and FS. These deficiencies were unknown in $40 \%$ of patients, mainly concerning falls, risk of depression, and FS, but only sporadically led to subsequent geriatric interventions. The reasons for this may be multiple, including the patient's wish and irrelevance owing to advanced stage of cancer, but in older patients without cancer, geriatric interventions have shown to improve the FS and survival. ${ }^{22}$ In the present study, the number of patients receiving geriatric interventions or a GA-adapted treatment regimen is too small to draw further conclusions on the impact of GA on outcome measures such as toxicity and functional decline. For this reason, intervention studies based on GA in older patients with CRC are needed in order to evaluate the impact of such personalized treatment on these different outcome measures.

Although the results of the GA were communicated to the treating physician before the final cancer treatment decision, we observed that only a minority of the physicians actually consulted them. This indicates that there is a need to educate physicians treating CRC on the benefits of GA. The International Society of Geriatric Oncology organizes such educational programs in order to train physicians (both cancer specialists and geriatricians) in the personalized care of older patients with cancer and to enhance cooperation with geriatric departments.

It is important for physicians to realize that FS is an important factor for quality of life in older patients and that active life expectancy is more important than life expectancy as such. ${ }^{23}$ In the present study, a decline in FS was observed in $18 \%$ for $\mathrm{ADL}$ and in $37 \%$ for IADL. A similar observation was made in surgically treated older patients with $\mathrm{CRC}$ where an $\mathrm{ADL}$ and $\mathrm{IADL}$ decrease was observed from the pre- to the postoperative condition. ${ }^{24}$ Similarly, the IADL decrease was the most pronounced, illustrating that more complex activities are affected to a higher degree than basic daily 
Table 4 Univariate and Multivariable Predictors of Functional Decline on ADL/IADL

\begin{tabular}{|c|c|c|c|c|c|c|c|c|c|}
\hline \multirow[b]{3}{*}{ Variable } & \multirow[b]{3}{*}{ Operationalization } & \multicolumn{4}{|c|}{ ADL Decline } & \multicolumn{4}{|c|}{ IADL Decline } \\
\hline & & \multirow{2}{*}{$\begin{array}{c}\text { Univariate } \\
P \text { Value }\end{array}$} & \multicolumn{3}{|c|}{ Multivariable } & \multirow{2}{*}{$\begin{array}{c}\text { Univariate } \\
P \text { Value }\end{array}$} & \multicolumn{3}{|c|}{ Multivariable } \\
\hline & & & $P$ Value & $\mathbf{O R}$ & $95 \% \mathrm{Cl}$ & & $P$ Value & $\mathbf{O R}$ & $95 \% \mathrm{Cl}$ \\
\hline Age, years & $70-74$ vs. $75-79$ vs. $\geq 80$ & .990 & & & & .832 & & & \\
\hline Timepoint of assessment & New diagnosis vs. progression/relapse & .590 & & & & .722 & & & \\
\hline Stage of disease & I-III vs. IV & .488 & & & & .771 & & & \\
\hline ECOG PS & Score $0-1$ vs. $2-4$ & .008 & - & & & .735 & & & \\
\hline fTRST & No geriatric profile vs. geriatric profile & .352 & & & & .376 & & & \\
\hline G8 & No geriatric profile vs. geriatric profile & .081 & - & & & .305 & & & \\
\hline Living situation & Living alone vs. living with help & .675 & & & & .706 & & & \\
\hline ADL & ADL independent vs. ADL dependent & .455 & & & & .769 & & & \\
\hline IADL & IADL independent vs. IADL dependent & .004 & .016 & 0.42 & $0.15-1.18$ & .544 & & & \\
\hline Falls history in the past 12 months & No falls vs. falls & .060 & - & & & .250 & & & \\
\hline Fatigue by MOB-T & No fatigue vs. fatigue & .005 & .066 & 0.37 & 0.13-1.04 & .350 & & & \\
\hline MMSE & $\begin{array}{l}\text { Normal cognition vs. mild/severe cognitive } \\
\text { decline }\end{array}$ & .023 & .136 & 0.37 & $0.10-1.41$ & .415 & & & \\
\hline GDS-15 & Not at risk vs. at risk for depression & .004 & .004 & 0.44 & $0.16-1.20$ & .307 & & & \\
\hline MNA-SF & $\begin{array}{l}\text { Normal nutritional status vs. at risk for } \\
\text { malnutrition/malnourished }\end{array}$ & .165 & - & & & .448 & & & \\
\hline $\mathrm{CCl}$ & No comorbidities vs. comorbidities & .909 & & & & .985 & & & \\
\hline Polypharmacy & $0-4$ different drugs vs. $\geq 5$ different drugs & .873 & & & & .262 & & & \\
\hline Surgery & No surgery vs. surgery & .599 & & & & .500 & & & \\
\hline Radiotherapy & No radiotherapy vs. radiotherapy & 208 & & & & .213 & & & \\
\hline Chemotherapy & No chemotherapy vs. chemotherapy & .613 & & & & .182 & - & & \\
\hline
\end{tabular}
No chemotherapy vs. chemotherapy

$P$ values in bold indicate those values significant to enter the multivariable regression for the univariate analysis $(P<.20)$ and to stay in the multivariable regression for the multivariable analysis $(P=.15)$.

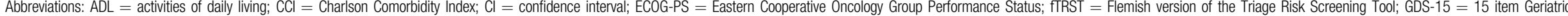
Depression Scale; IADL $=$ instrumental activities of daily living; MMSE $=$ Mini Mental State Examination; MNA-SF $=$ Mini Nutritional Assessment - Short Form; MOB-T $=$ Mobility - Tiredness Test; OR $=$ odds ratio 
Table 5 Univariate and Multivariable Predictors of Grade $3 / 4$ Hematologic and Nonhematologic Chemotherapy-Related Toxicity

\begin{tabular}{|c|c|c|c|c|c|c|c|c|c|}
\hline \multirow[b]{3}{*}{ Variable } & \multirow[b]{3}{*}{ Operationalization } & \multicolumn{4}{|c|}{ Grade 3/4 Hematologic Toxicity } & \multicolumn{4}{|c|}{ Grade 3/4 Nonhematologic Toxicity } \\
\hline & & \multirow{2}{*}{$\begin{array}{c}\text { Univariate } \\
\boldsymbol{P} \text { Value }\end{array}$} & \multicolumn{3}{|c|}{ Multivariable } & \multirow{2}{*}{$\begin{array}{c}\text { Univariate } \\
\boldsymbol{P} \text { Value }\end{array}$} & \multicolumn{3}{|c|}{ Multivariable } \\
\hline & & & $P$ Value & OR & $95 \% \mathrm{CI}$ & & $P$ Value & $\mathbf{O R}$ & $95 \% \mathrm{Cl}$ \\
\hline Age, years & $70-74$ vs. $75-79$ vs. $\geq 80$ & .313 & & & & .187 & - & & \\
\hline Timepoint of assessment & New diagnosis vs. progression/relapse & .108 & - & & & .934 & & & \\
\hline Stage of disease & I-III vs. IV & .025 & .040 & 6.6 & $1.09-39.8$ & .310 & & & \\
\hline ECOG-PS & Score $0-1$ vs. $2-4$ & .237 & & & & .148 & & & \\
\hline fTRST & No geriatric profile vs. geriatric profile & .740 & & & & .831 & & & \\
\hline G8 & No geriatric profile vs. geriatric profile & .102 & - & & & .084 & - & & \\
\hline Living situation & Living alone vs. living with help & .697 & & & & .505 & & & \\
\hline ADL & ADL independent vs. ADL dependent & .810 & & & & .214 & .087 & 2.62 & $0.86-7.97$ \\
\hline IADL & IADL independent vs. IADL dependent & .936 & & & & .934 & & & \\
\hline Falls history in the past 12 months & No falls vs. falls & .935 & & & & .625 & & & \\
\hline Fatigue by MOB-T & No fatigue vs. fatigue & .040 & .067 & 8.2 & $0.87-76.8$ & .043 & .120 & 0.30 & 0.09-1.01 \\
\hline MMSE & $\begin{array}{l}\text { Normal cognition vs. mild/severe } \\
\text { cognitive decline }\end{array}$ & .794 & & & & - & & & \\
\hline GDS-15 & Not at risk vs. at risk for depression & .890 & & & & .528 & & & \\
\hline MNA-SF & $\begin{array}{l}\text { Normal nutritional status vs. at risk for } \\
\text { malnutrition/malnourished }\end{array}$ & .065 & - & & & .026 & .026 & 0.33 & $0.09-1.27$ \\
\hline $\mathrm{CCl}$ & No comorbidities vs. comorbidities & .328 & & & & .335 & & & \\
\hline Polypharmacy & $0-4$ different drugs vs. $\geq 5$ different drugs & .337 & & & & .376 & & & \\
\hline
\end{tabular}

$P$ values in bold indicate those values significant to enter the multivariable regression for the univariate analysis $(P<.20)$ and to stay in the multivariable regression for the multivariable analysis $(P=.15)$.

of the Triage Risk Screening Tool; GDS-15 = 15 item Geriatric Depression Scale; IADL = instrumental activities of daily living; MMSE = Mini Mental State Examination; MNA-SF = Mini Nutritional Assessment - Short Form; MOB-T = Mobility - Tiredness Test; OR = odds ratio. 


\section{Geriatric Assessment in Colorectal Cancer}

functions. In a systematic review in patients treated for CRC with curative intent, FS declined at 3 months after treatment and improved again over time, although it was not fully back to baseline at 1 year. ${ }^{25}$ Interestingly, a significant number of patients experienced a permanent FS decrease $31 \%$ for ADL and $61 \%$ for IADL at 22 months). The identification of predictive factors for FS decline could guide treatment and interventions in order to prevent such functional decline and consequently preserve quality of life. In our study, baseline IADL, fatigue, depression, and cognition were found to be predictive for $\mathrm{ADL}$ decline at 2 to 3 months, whereas no predictive factors were identified for IADL decline. Interestingly, treatment with chemotherapy was not predictive for FS decline in our population.

Grade 3/4 chemotherapy-related toxicity occurred in a quarter of patients treated with chemotherapy, mostly nonhematologic. Baseline malnutrition, fatigue, and ADL were predictive for grade $3 / 4$ nonhematologic toxicity, whereas stage and baseline fatigue were predictive for grade $3 / 4$ hematologic toxicity. Because patients with advanced stage (III and IV) significantly received systemic treatment more frequently, these patients were more likely to develop hematologic toxicity. In other studies in older patients with CRC, malnutrition, cognition, and IADL were mentioned as predictive factors for chemotherapy-related toxicity. ${ }^{10,26}$ In addition, GA seems to be predictive for treatment-related toxicity, ${ }^{20,21,27}$ postoperative morbidity, ${ }^{9}$ and decrease in quality of life. ${ }^{8}$ This illustrates the relevance of GA in the treatment decision-making process.

One important limitation of this study is the heterogeneity of the population with CRC regarding stage and treatment. In addition, because the current study was performed at 2 Belgian academic centers, a referral bias may be present. Therefore, it may be difficult to apply our exact results to the overall populations of older patients with CRC. In order to investigate the full impact of GA and geriatric interventions, a randomized clinical trial in a larger more homogeneous population would be necessary.

\section{Conclusion}

In conclusion, the treatment of older patients with CRC requires careful consideration of multiple factors by the treating physician. An evaluation by a GA will help to identify patients at risk for adverse outcome and will help to develop a treatment plan incorporating medical and physical considerations, cancer- and comorbidity-related outcomes, and the patient's health and treatment goals. In the absence of risk factors, GA may also prevent undertreatment in fit patients. For this reason, integration of GA in routine clinical practice is of the utmost importance. To obtain this goal, the International Society of Geriatric Oncology organizes educational programs for all caregivers involved in the treatment of older patients with cancer as well as for geriatricians in an order to provide these physicians with the necessary skills to treat older patients with cancer multidisciplinary and to facilitate reciprocal cooperation. $^{28}$

\section{Clinical Practice Points}

- Although fit older patients with CRC may derive benefit from surgery and chemotherapy, chronologic age is a frequent motivation for the omission of therapy. For this reason, it has been stated that biologic age as determined by GA should be used to decide if a patient is fit or not to undergo treatment.

- The current study demonstrates that a GA is abnormal in a substantial amount of older patients with CRC, even in the subgroup with good ECOG PS, and frequently detects previously unknown problems. However, only a small percentage of treating physicians actively consulted the results, and the impact on the performance of directed interventions and on treatment decisions remained limited. The study also demonstrates that functional decline occurs frequently in older patients with CRC during treatment and that GA parameters may be predictive for functional decline and chemotherapy-related toxicity.

- These results clearly indicate the need to educate treating physicians on the usefulness of GA. Increasing cooperation between geriatricians and physicians treating CRC will lead to a more personalized therapy and directed interventions for older patients and subsequently should prevent functional decline and chemotherapy-related toxicity.

\section{Acknowledgment}

This work was supported by the Vlaamse Liga Tegen Kanker.

\section{Disclosure}

The authors have stated that they have no conflicts of interest.

\section{References}

1. Papamichael D, Audisio RA, Glimelius B, et al. Treatment of colorectal cancer in older patients: International Society of Geriatric Oncology (SIOG) consensus recommendations, 2013. Ann Oncol 2015; 26:463-76.

2. Lewis JH, Kilgore ML, Goldman DP, et al. Participation of patients 65 years of age or older in cancer clinical trials. J Clin Oncol 2003; 21:1383-9.

3. Colorectal Cancer Collaborative Group. Surgery of colorectal cancer in elderly patients: a systematic review. Lancet 2000; 356:968-74.

4. Hamaker ME, van Rixtel B, Thunnissen P, et al. Multidisciplinary decisionmaking on chemotherapy for colorectal cancer: an age-based comparison. $J$ Geriatr Oncol 2015; 6:225-32.

5. Wedding U, Kodding D, Pientka L, et al. Physicians' judgement and comprehensive geriatric assessment (CGA) select different patients as fit for chemotherapy. Crit Rev Oncol Hematol 2007; 64:1-9.

6. Solomon D, Sue Brown A, Brummel-Smith K, et al. National Institutes of Health Consensus Development Conference Statement: geriatric assessment methods for clinical decision-making. J Am Geriatr Soc 2003; 51:1490-4.

7. Kenis C, Bron D, Libert Y, et al. Relevance of a systematic geriatric screening and assessment in older patients with cancer: results of a prospective multicentric study. Ann Oncol 2013; 24:1306-12.

8. Wildiers H, Heeren P, Puts M, et al. International Society of Geriatric Oncology consensus on geriatric assessment in older patients with cancer. J Clin Oncol 2014; 32:2595-603.

9. Kristjansson SR, Nesbakken A, Jordhoy E, et al. Comprehensive geriatric assessment can predict complications in elderly patients after elective surgery for colorectal cancer. A prospective observational cohort study. Crit Rev Oncol Hematol 2010; 76:208-17.

10. Aaldriks AA, van der Geest LG, Giltay EJ, et al. Frailty and malnutrition predictive of mortality risk in older patients with advanced colorectal cancer receiving chemotherapy. J Geriatr Oncol 2013; 4:218-26.

11. Decoster L, Van Puyvelde K, Mohile S, et al. Screening tools for multidimensional health problems warranting a geriatric assessment in older cancer patients: an update on SIOG recommendations. Ann Oncol 2015; 26:288-300.

12. Kenis C, Decoster L, Van Puyvelde K, et al. Performance of two geriatric screening tools in older patients with cancer. I Clin Oncol 2014; 32:19-26.

13. Bellera CA, Rainfray M, Mathoulin-Pélissier S, et al. Screening older cancer patients: first evaluation of the G-8 geriatric screening tool. Ann Oncol 2012; 23: 2166-72.

14. Division of Cancer Treatments and Diagonsis, National Cancer Institute. Cancer Therapy Evaluation Program. Available at: www.ctep.cancer.gov. Accessed: September 6, 2016.

15. Decoster L, Kenis C, Van Puyvelde K, et al. The influence of clinical assessment (including age) and geriatric assessment on treatment decisions in older cancer patients. J Geriatr Oncol 2013; 4:235-41.

16. Shrag D, Cramer LD, Bach PB, et al. Age and adjuvant chemotherapy use after surgery for stage III colon cancer. J Natl Cancer Inst 2001; 93:850-7. 
17. Dotan E, Browner I, Hurria A, et al. Challenges in the management of older patients with colon cancer. I Natl Compr Cancer Netw 2012; 10:213-25.

18. Jolly TA, Deal AM, Nyrop K, et al. Geriatric assessment-identified deficits in older cancer patients with normal performance status. Oncologist 2015; 20: 379-85.

19. Pope D, Ramesh H, Gennari R, et al. Pre-operative assessment of cancer in the elderly (PACE): a comprehensive assessment of underlying characteristics of elderly cancer patients prior to elective surgery. Surg Oncol 2006; 15:189-97.

20. Extermann M, Boler I, Reich RR, et al. Predicting the risk of chemotherapy toxicity in older patients: the Chemotherapy Risk Assessment Scale for High-Age Patients (CRASH) score. Cancer 2012; 118:3377-86.

21. Hurria A, Togawa K, Mohile SG, et al. Predicting chemotherapy toxicity in oldet adults with cancer: a prospective multicenter study. J Clin Oncol 2011; 29:345765.

22. Stuck AE, Siu AL, Wieland GD, et al. Comprehensive geriatric assessment: a metaanalysis of controlled trials. Lancet 1993; 342:1032-6.
23. Chouliara Z, Miller M, Stott D, et al. Older people with cancer: perceptions and feelings about information, decision-making and treatment - a pilot study. Eur J Oncol Nurs 2004; 8:257-61.

24. Ronning B, Wyller TB, Jordhoy MS, et al. Frailty indicators and functional status in older patients after colorectal cancer surgery. I Geriatr Oncol 2014; 5:26-32.

25. Hamaker ME, Prins MC, Schiphorst AH, et al. Long-term changes in physical capacity after colorectal treatment. J Geriatr Oncol 2015; 6:153-64.

26. Aparicio T, Jouve JL, Teillet L, et al. Geriatric factors predict chemotherapy feasibility: ancillary results of FFCD 2001-02 phase III study in first line chemotherapy for metastatic colorectal cancer in elderly patients. J Clin Oncol 2013; 31:1464-70.

27. Kurniali PC, Hrinczenko B, Al-Janadi A. Management of locally advanced and metastatic colon cancer in elderly patients. World J Gastroenterol 2014; 20:191022.

28. International Society of Geriatric Oncology. Available at: www.siog.org. Accessed: September 6, 2016. 